\title{
Lived Experiences of Survivors of COVID-19 Disease at Hwange Colliery Hospital, Zimbabwe
}

\author{
Joshua Munsaka, Judith Rukweza
}

\section{ABSTRACT}

The COVID-19 pandemic is one of the worst global nightmares in the past decade for both healthcare workers and general public. The initial focus has been on the lifesaving and biomedical aspects, but COVID-19 has rapidly established itself as an illness that negatively impacts the psychosocial health of all affected individuals. The purpose of this study was to explore the lived experiences of survivors of COVID-19 disease with intent to identify gaps within the healthcare system in responding to the COVID-19 pandemic and therefore improve the patient care. A phenomenological approach was used and a purposive sampling technique employed to select 15 participants at Hwange Colliery hospital for the study. All the participants were individuals aged between 19 and 55 years who had recovered from COVID-19 disease. Participants were either Hwange Colliery Company Limited employees or dependents and resident of Hwange Colliery concession area. Analysis of data was done during data collection using the Braun and Clarke (2006) thematic analysis. Three themes with eleven subthemes emerged from data and these were: awareness of COVID-19 disease; sources of information and trust of sources of information, causes and origins of the COVID-19, health seeking; physical manifestations, specimen collection and experience, treatment and home remedies, interaction with healthcare workers, psychosocial dimension; isolation and experiences, fears and concerns, family and friends support, religious, job security. The study revealed the lived experiences of survivors of COVID-19 disease. The survivors' fear and coping mechanisms were made plain. The age of participants ranged from 19 years to 55 years with a mean age of 36.3 and a standard deviation of 5.1.

Submitted : September 29, 2021

Published : December 13, 2021

ISSN: $2593-8339$

DOI: $10.24018 /$ ejmed.2021.3.6.1084

\section{J. Munsaka*}

Hwange Colliery Hospital School of Nursing, Hwange, Zimbabwe.

(e-mail: munsakajoshua@gmail.com)

\section{J. Rukweza}

University of Zimbabwe, Faculty of Medicine and Health Sciences, Department of Primary Health Care Sciences, Nursing Science Unit, Avondale, Harare, Zimbabwe.

(e-mail: rukwezajudith@gmail.com)

*Corresponding Author

Keywords: Coronavirus, COVID-19 survivor, lived experiences, SARS- CoV2.

\section{INTRODUCTION}

Like veld fire, there has been a continuous rapid spread of COVID-19 worldwide. Since the beginning of the outbreak, in December 2019 up to 2 June 2021, over 172 million cases have been confirmed in more than 200 countries and deaths were around 3.5 million deaths [1]. The COVID-19 pandemic is one of the worst nightmares in the past decade for both healthcare workers and the general public. A number of lessons have been drawn by countries from this pandemic.

COVID-19 is a severe blow to the healthcare system of the entire world and has affected millions of people across the world [2]. COVID-19 is associated with a very high rate of infectivity, which has led to a high level of fear and anxiety of getting infected. There has been less focus on the lived experiences of COVID-19 Survivors, since this is an emerging disease [3].

The initial focus of research has been on the lifesaving and biomedical aspects, but COVID-19 has rapidly established itself as an illness that negatively impacts the psychosocial health of all affected individuals [4]. Public health specialists all around the world are calling for a shift in focus and attention to the need to study the lived experience and behaviour of individuals during this outbreak [5].

The burden of the disease has been overwhelming especially on healthcare system including the healthcare workers who are in the forefront fighting the disease. With this, it is possible to pay less attention at what the patients who test positive to COVID-19 go through. Most researchers are arguably toiling hard for an effective antiviral and vaccine for elimination of COVID-19. Due to this focus, there is a tendency to view pandemics such as this, as a medical phenomenon only resulting in neglect on other dimensions such as mental health and social issues [6]. COVID-19 disease is associated with high rate of infectivity and as such brings with it fear and anxiety [2]. The World Health Organization has emphasized on the need to consider mental health for both patients and healthcare workers involved in the care of COVID-19 patients [7]. As reported in [2] and [6], anxiety, excessive worries, irritability, low mood, frustration, distress, loneliness are major mental health concerns for the patients with COVID-19 disease. This warranty the inclusion and active participation of mental health specialists such as nurses (trained in mental health) and psychiatrist right from diagnosis to recovery of the patients. 


\section{Methods And Materials}

This study utilized the interpretive phenomenological approach. The study was conducted at Hwange Colliery Hospital. The hospital is located in the mining town of Hwange in Zimbabwe. Hwange Colliery Hospital is a teaching hospital which is privately owned by Hwange Colliery Company Limited (HCCL) and caters largely the Hwange urban population. A purposive sampling approach was used in recruiting 15 participants for the face to face interviews. The age range of participants was 19 to 55 years. The inclusion criteria were having a positive PCR COVID19 test result whether symptomatic or asymptomatic at diagnosis time, the participants had recovered or discharged from self-isolation. Individuals who were mentally unable to sustain conversation and or give coherent and meaningful responses were excluded from the study.

Approval for the study was obtained from the study site, Joint Research Ethics Committee (JREC/115/2021) and Medical Research Council of Zimbabwe (MRCZ/B/2150). The instrument was designed by the first author and reviewed by second author and other experts in the field. The interview guide was pretested looking at sequence and terminology used in the study protocol. Both verbal and written consent was obtained from each participant after explanation of the study intent. The interviews duration ranged between 26 and 57 minutes. Bracketing was used to avoid bias. The first author repeatedly listened to the audio recordings and read the transcriptions over and over to exclude preconceptions of the researcher. Data collection was done and discontinued at data saturation, when no additional data could be obtained from the interviews. The data was analyzed using Braun and Clarke [8].

\section{RESULTS}

The study explored the lived experiences of survivors of COVID-19 disease at Hwange Colliery Hospital. The researcher conducted in-depth interviews with survivors of COVID-19 disease to understand their experiences from their own point of view. The 15 participants were coded as one to fifteen.

\section{A. Demographic Characteristics}

Table I below shows the demographic characteristics of study participants. As shown above, seven (47\%) were males and eight (53\%) females participated in the study. Regarding age, the youngest participant was a 19 year old, while the oldest was 55 years old. The mean sample age was 36.3 with a standard deviation of 5.1 . Eleven $(73 \%)$ participants had no underlying conditions while 4 (27\%) were all hypertensive patients on medication.

\section{B. Data Explication and Presentation of Findings}

There were three main themes with eleven subthemes, which emerged from the data, and these are presented as awareness of COVID-19 disease, health seeking behavior and psychosocial dimension.
TABLE I: DEMOGRAPHIC CHARACTERISTICS OF PARTICIPANTS

\begin{tabular}{ccccc}
\hline \multicolumn{2}{c}{ Variable } & $\begin{array}{c}\text { Survivors } \\
(\mathrm{n}=15)\end{array}$ & Percentage & $\begin{array}{c}\text { Total } \\
(\%)\end{array}$ \\
\hline \hline \multirow{2}{*}{ Sex } & Male & 7 & 46.7 & 100 \\
& Female & 8 & 53.3 & \\
Age & $18-39$ & 10 & 66.7 & 100 \\
Race & $40-60$ & 5 & 33.3 & 100 \\
Marital & African & 15 & 100 & 100 \\
status & Single & 6 & 40 & 100 \\
Level of & Married & 9 & 60 & 100 \\
education & Secondary & 5 & 33.3 & \\
Religion & Tertiary & 10 & 66.7 & 100 \\
& Christian & 12 & 80 & \\
Residence & Traditional & 3 & 20 & \\
& Township & 9 & 60 & \\
& Lwendulu & 5 & 33.3 & \\
\hline \hline
\end{tabular}

Source: researcher's findings.

\section{Awareness of COVID-19 Disease}

The theme awareness of COVID-19 included the causes and origin of disease, sources and trust of source of information on COVID-19. Nine (60\%) participants mentioned the television with various channels including ZBC TV, SABC, Aljazeera, and BBC.

The social media such as Whatsapp mentioned by 10 $(67 \%)$ and Facebook reported by $5(33 \%)$ are some of their main source of information on COVID-19.

"I used to get the Hwange Colliery Company weekly bulletin specifically on COVID-19. I also watched our local national broadcaster (ZBC TV) and South African Broadcasting Corporation (SABC). There were also messages circulating on social media platforms especially Whatsapp." (Participant 2)

While the Hwange Colliery provided the COVID-19 Weekly magazine, some employees never bothered to read the magazine at their disposal as they had told themselves that they were immune to the disease as shown in the extract below.

"At workplace there was a weekly COVID-19 bulletin that I cared less to read. I had told myself that I will not catch the disease." (Participant 13)

Regarding how participants trusted their sources, all acknowledged that information from the television was better trusted than social media platforms.

\section{Health Seeking Behaviour}

This theme included physical manifestations, diagnostic testing and experiences as well as treatment and home remedies. Symptoms reported ranged from sore throat and flu, headache, general body weakness, general body pains, dry cough, shortness of breath on exertion and at rest, diarrhoea and vomiting. Seven participants (47\%) reported shortness of breath while $6(40 \%)$ experienced a headache which ranged from moderate to severe. Nausea and vomiting, and chills were each reported by four (27\%) participants. Chest pain and fatigue was each experienced by three participants. Four (27\%) participants reported loss of appetite, and loss of senses of smell and taste. Two (13\%) participants reported diarrhoea while another two (13\%) experienced dry cough.

For pregnancy and childbearing, the symptoms of COVID19 were confused with effects of pregnancy or puerperium as shown in the following extracts. 
"I was having shortness of breath. I was pregnant, and advanced almost due, and the symptoms for me only suggested that they were due to pregnancy. " (Participant 11)

Some participants described symptoms that persisted over longer period than anticipated by the survivors.

"I had a sore throat, I wondered what kind of a sore throat it was, all I can say was that it was different, unfamiliar and stubborn, it just would not go away. The sore throat never worsened but it lingered for long and remained moderate." (Participant 3).

"I usually experience migraine headaches, but this headache was different, unfamiliar, deep seated and lingered longer." (Participant 15).

The time taken to report at a health facility is influenced by a number of factors. The researcher only explored how much time was taken by participants to report to a health facility from onset of symptoms. Most participants took four days to report to the healthcare facility for health services.

"On day 4, I went to the hospital, when I realized that the symptoms were not subsiding." (Participant 7).

The gold standard for the diagnostic test is the Polymerase Chain Reaction (PCR) which requires a nasopharyngeal and an oropharyngeal specimen and all the fifteen participants had the PCR test done. The experience of specimen collection was described by the participants in diverse views as some described it as a horrible experience while others described it as an irritating procedure.

"I had specimen collected from nose and another from mouth and was irritating but manageable, not as exaggerated by people." (Participant 14).

The time taken to receive the results varied greatly from a minimum of 2 hours to about 6 days waiting time. All participants disclosed the positive COVID-19 result to close family members (father, mother, husband, wife, children, or any person living in the same house), as well as supervisors at work (for employees).

"For disclosure of COVID-19 results, I informed my sister and my husband." (Participant 2).

Some participants cried upon receiving the positive COVID-19 test results. This, they did to express their fears and shock at the diagnosis.

"When I got the news of positive results, I informed my husband, and I started crying while on the phone, told him I have a diagnosis of a disease from the TV. I thought I was dying, that is why I was crying." (Participant 10).

For treatment, common among participants was the use of painkillers such as paracetamol, codeine and tramacet and antibiotics such as amoxicillin, azithromycin, steroids such as prednisolone, and cough mixtures as well as supplements such as vitamin $\mathrm{C}$, and zinc sulphate. The common herbs were ginger, garlic and lemon which was used by $9(60 \%)$ participants and zumbani tea reported by 5 (33\%) participants. Some herbs were added to tea (zumbani, garlic, guavas, ginger, lemon, and cinnamon) while others were added to water for steaming (tsunami and eucalyptus oil).

"There were many concoctions prepared using different recipes, which we got from friends. The list included zumbani, garlic, ginger, lemon, guavas. We used to have tea from these herbs twice a day. Moreover we used to steam twice a day." (Participant 4).
For some participants, the home remedies were not effective and did not bother to use them.

"They tried to give me the home remedies, the zumbani, lemon and they also tried steaming, but this did not work. I do not believe in those home remedies, I think it's a mental thing." (Participant 7).

Most participants reported having received the expected care and interacted well with healthcare workers. However some participants reported having observed obvious signs of fear among healthcare workers.

"When I explained the symptoms to the doctor, he promised to give me medication, but he went away and never came back. I could call the nurse and they never came to assist me. Nurses like other health workers were scared. People were scared, hence the hesitancy to attend to me." (Participant 6).

\section{E. Psychosocial Dimension}

The lived experiences on self-isolation varied greatly among participants. Two (13\%) participants were at some point transferred to the COVID-19 isolation centre and later completed self-isolation while at home. Thirteen (87\%) participants were sent home for self-isolation.

"I was sent home for self-isolation since the symptoms were not very bad. However the situation changed when I was at home, I became worse, thank God the Case Management Team used to check on me daily, and discovered I was very sick. I was immediately moved to the COVID-19 isolation centre where I received oxygen per face mask. I was later discharged to complete self-isolation at home." (Participant 15).

Some participants had vowed not to be cared for in the COVID-19 isolation centre which they described as a no go area.

"For me, the COVID-19 isolation centre was a no go area, I have been to the place, I know it, and it is deserted, no entertainment, no I cannot go there, what will I be doing there for 14 days." (Participant 4).

The isolation centre was well suited for very ill patients and not for those who have recovered awaiting discharge.

"I was too sick therefore I never worried about the isolation centre initially. Later, when I started recovering, there was silence, and I became bored. I think to cure the boredom; the centre should provide a radio or television." (Participant 6).

Self-isolation presented its problems such as food procurement as some individuals thrive on hand to mouth on daily basis and as such confinement to the house limits their hustling for survival.

"When I was discharged from the COVID-19 isolation centre, I came home to complete self-isolation. We had one problem-no food-this forced me to go out and look for food." (Participant 6).

"I was on self-isolation at home and spent time with teenagers. We would quarrel, sometimes my son would explode to say, I do not have COVID-19, so why should I be confined? This is house arrest." (Participant 12).

Family and friends play an important role in times of illness by providing care to affected members. Psychological support is very important in managing the patient. The support is shown in the extracts below. 
"We got support from our friends, we got bread on a daily basis, friends would ask us if we needed anything from the shops, then they would call once they are at the gate to come and collect bought items." (Participant 4).

Participants reported fear concerning the infection. Six $(40 \%)$ reported fear of death and its consequences such as care of the children left behind. Three $(20 \%)$ participants were afraid of spreading the disease to their loved ones. Uncertainty was experienced for varying reasons and factors. The majority of participants $12(80 \%)$ were uncertain of the progression of the disease over time.

"When I received the results, I thought the whole world was coming to an end for me, hence I cried. I was worried about who was going to take care of my child if I died." (Participant 10).

Pregnancy presented more uncertainty especially with midwives unsure on how to handle the COVID-19 positive pregnant women.

"I was pregnant and had gone for ANC hoping to have an ultrasound scan to check the position of my baby in the womb. The nurses told me that they would not attend to me while on self-isolation. What if time for delivery comes, how will I be assisted during delivery?" (Participant 11).

Two (13\%) participants were uncertain on what will happen to their employment, will it be terminated or not. "It was difficult for me to tell my supervisor at work. He had to call me, when he realized I was absent first day after leave. I gathered courage to inform him, when I did his response was, It's fine, there is no need to come to work until your selfisolation is over. I was uncertain of my supervisor's response. If I informed him, maybe he would terminate my contract- I was afraid." (Participant 11).

Below is Table II showing summary of themes and subthemes that emerged from data analysis.

TABLE II: THEMES AND SUBTHEMES EMERGING FROM DATA

\begin{tabular}{|c|c|c|}
\hline $\begin{array}{l}\text { Theme } \\
\text { Number }\end{array}$ & Theme category & Subthemes \\
\hline 1 & $\begin{array}{c}\text { Awareness of } \\
\text { COVID-19 disease }\end{array}$ & $\begin{array}{l}\text { Sources of information and trust } \\
\text { of the sources } \\
\text { Causes and origin of COVID-19 }\end{array}$ \\
\hline 2 & $\begin{array}{l}\text { Health seeking } \\
\text { behaviour }\end{array}$ & $\begin{array}{c}\text { Physical manifestations } \\
\text { Time taken to present at health } \\
\text { facility } \\
\text { Specimen collection and } \\
\text { experiences }\end{array}$ \\
\hline & & $\begin{array}{l}\text { Treatment and home remedies } \\
\text { Interaction with health workers }\end{array}$ \\
\hline 3 & $\begin{array}{l}\text { Psychosocial } \\
\text { dimension }\end{array}$ & $\begin{array}{l}\text { Fears and concerns } \\
\text { Family and friends support } \\
\text { Job security } \\
\end{array}$ \\
\hline
\end{tabular}

Source: researcher's findings

\section{Discussion}

The age of participants ranged from 19 years to 55 years with a mean age of 36.3 and a standard deviation of 5.1 . Eleven $(73 \%)$ participants had no underlying conditions while $4(27 \%)$ were all hypertensive patients on medication. Underlying conditions place individuals at severe disease with complications. In a study by Sahoo et al., all those who were admitted in Intensive Care Unit had underlying disease, the majority of them with Diabetes Mellitus [3]. In this study, the two survivors who were admitted at the COVID-19 isolation centre were all on treatment for hypertension that possibly led to severe symptoms. According to WHO, [7] there are several factors that predispose to serious illness; these include old age, presence of underlying illness such as diabetes mellitus and cancer.

The results from the study show that all participants were aware of COVID-19 as a global disease that has affected world population and that it originated from China. The main sources of information on COVID-19 was the social media platform (WhatsApp) (67\%) followed by television (60\%). The primary sources of information included radio and television [9]. In a study at the New York University, about ninety percent had the television, radio and newspapers as the sources of information on COVID-19 disease outbreak [10]. In the same study $43.3 \%$ of study participants trusted the government controlled website as source of authentic information regarding COVID-19 disease. Social media also plays an essential role in information dissemination as reported in same study where $73.6 \%$ used social media as the source of information on COVID-19 disease [10].

In this study $13(87 \%)$ participants presented with various symptoms of different severity and 2 (13\%) were asymptomatic. The symptoms included shortness of breath (47\%), headache (40\%), nausea and vomiting (27\%) loss of appetite (27\%), diarrhoea (13\%). Previous studies, suggest that headache, loss of sense of smell and taste, loss of appetite, nausea vomiting, and diarrhoea, fever, cough, and shortness of breath are common among COVID-19 infected patients [2], [11], [12]. The symptoms, though common, were reported as different, unfamiliar and unusual. Findings in this study show that the symptoms were different and lingered for long and described as stubborn not responding to common medication. Some COVID-19 patients experience complications that may stretch for several months post initial recovery, which have come to be called Long-COVID or COVID long-haulers [13]. Some participants described the symptoms as those similar to malaria presentation. This could be attributed to the fact that Hwange is a malaria prone area, and as such malaria symptoms are more common to the residents than for other diseases.

Some individuals thought that the disease was not meant for Africans but only for other races. This is almost similar to what previous studies reported where it is acknowledged that most people generally believe that negative events such as becoming ill to being involved in an unfortunate incident should happen to others than to them [14], [15].

The specimen collection from the nose and mouth was described by some participants as painful or horrible while some participants described it as just some discomfort procedure. This was consistent with findings from previous study where participants reported being uncomfortable with nasopharyngeal swab collection [11].

All the 15 participants disclosed to the family (parents, siblings and children and anyone they were staying together). Some informed friends (60\%), neighbours (7\%) and church members $(27 \%)$. According to these findings, the neighbours were least informed, while shockingly the church members were not also popular on the disclosure list. This may depict how these social institutions are valued by individuals in times of stressful event. The order of how the people were informed may suggest the order of priority in someone's life. 
The majority of participants resorted to home remedies chief among them was ginger, garlic and lemon accounting for $60 \%$, zumbani $33 \%$ - added to tea while tsunami and eucalyptus were added to hot water for steaming. The use of lemon, herbal tea, multivitamins, honey and cough syrup helped to alleviate symptoms in the participants from previous studies [11], [12].

While most participants reported satisfaction with their interaction with healthcare workers, one $(7 \%)$ participant complained of having been neglected. In previous studies, health workers have reported minimized contact time with COVID-19 patients to prevent possibility of contracting the deadly viral disease [9], [11]. The fear exhibited by clinicians when attending to Ebola patients where they maintained safe distance and reduced contact time as a measure of minimizing the possibility of contracting the deadly virus was reported in previous studies [16].

Following onset of symptoms some participants reported for health services within 24 hours while others took the wait and see attitude commonly exhibited by populations. This is contrary to what was reported by [11] that participants delayed seeking treatment as they viewed hospitals as death trap.

The experiences while on self-isolation at home was marked by spending time on watching the television, surfing the internet for some participants, while others were on social media platforms such as WhatsApp and Facebook. For one (7\%) participant, it was an opportunity to get closer to the maker, followed online church services and reflected on the relationship with God. It is difficult to spend the day doing nothing when one is used to be occupied throughout the day. Social isolation, distress, boredom and frustration are related to confinement, abnormally reduced social and physical contact with others as well as loss of usual habits [17], [18].

Hwange Colliery's Case Management Team (CMT) has included a mental health nurse in each team for the purpose of counseling in case of need situation during the care of COVID-19 patients. The inclusion and active participation of mental health specialist is supported in previous studies [2], [6]. Their (mental health nurses) active participation would help the COVID-19 patients cope with the illness and face friends and workmates who may have divergent views regarding COVID-19.

Fear remains the greatest concern among patients who are diagnosed with COVID-19 disease. As reported in this study, some participants had experienced sudden change in their health status during the course of their self-isolation period. In previous studies, patients with a diagnosis of COVID-19 worried about deterioration of symptoms over time [12], [19]. Four $(27 \%)$ participants reported fear of infecting family members, a finding which is consistent with results from previous studies, on both current and past pandemics [3], [11], [20]. In this study, findings show that the pregnant woman was worried about infecting the unborn child and the breastfeeding women were worried about infecting their babies during breastfeeding. In a relatively comparative study, [21] in their study on 2009 H1N1 influenza pandemic reported almost similar results. However [22] in a systematic scoping review notes that there is no evidence at the moment that suggests vertical transmission or a different disease pattern in pregnant women than in general population.
Uncertainty about job security was among the factors inducing fear among participants. Most jobs have been lost across the globe due to COVID-19. Some contracts have been terminated due to restrictions imposed by governments to stop the spread of COVID-19. In one study worry over job loss was one of the factors contributing to fear among employed participants [12].

\section{RECOMMENDATIONS}

The study recommends the following:

Regardless of symptoms, the community should be informed through campaigns to seek treatment early so as to prevent continued exposure of their loved ones and the public at large from the infected individuals.

Counseling to all patients that are distressed by the diagnosis of COVID-19 disease should be done. This will enable the patients to cope with stigma associated with COVID-19.

Refresher training of nurses and midwives as well as other health workers regarding Infection Prevention and Control measures such as proper donning and doffing, so that this minimizes the chances of spread of infection to health workers.

Research is recommended to ascertain the effectiveness and risks of herbal tea which is common home remedies revealed in this study.

The COVID-19 isolation centres should accommodate entertainment, as clients admitted there become lonely or bored owing to less time spent by health workers for obvious reasons of reducing exposure time and therefore minimize the possibility of contracting infection by the health workers.

The social worker should be engaged as part of multisectoral approach in addressing the needs of the patients such as basic needs like food acquisition.

\section{CONCLUSION}

Fear due to uncertainty of disease progression and fear of death were among the chief concerns forwarded by the survivors and must be addressed to enable early recovery. Safety and health talks are essential to employees as they must adopt them not only their safety but that everyone safety is guaranteed. Continuous training of health workers such nurses and midwives is mandatory to enable them to handle patients who are highly infectious. The inclusion of mental health specialists into the management of COVID-19 patients and their families can never be overemphasized at this point as it plays a pivotal role in reduction of fear and uncertainty thereby promoting early recovery of patients.

\section{ACKNOWLEDGMENT}

The authors wish to acknowledge the participants who shared valuable experiences for the study.

\section{REFERENCES}

[1] World Health Organization. Coronavirus. [Internet] [cited on: 2021 April 2] Available from: https://www.who.int/healthtopics/coronavirus\#tab=tab_1 
[2] Sahoo S, Mehra A, Suri V, Malhotra P, Yaddanapudi NL, Puri GD, et al. Lived experiences of corona survivors (patients admitted in COVID wards): A narrative real-life documented summaries of internalized guilt, shame, stigma, anger. Indian Journal of Psychiatry. 53; 2020a.

[3] Sahoo S, Mehra A, Suri V, Malhotra P, Yaddanapudi NL, Puri GD, et al. Lived Experiences of Covid-19 Intensive Care Unit Survivors. Indian Journal of Psychological Medicine. 42: 4387-390; 2020b.

[4] Haider I, Tiwana F, Tahir SM, Impact of the COVID-19 Pandemic on Adult Mental Health. Pakistan Journal of Medical Sciences. 2020; 36(4):2756.

[5] Sun N, Shi S, Jiao D, Song R, Ma L, Wang H. A qualitative study on the psychological experience of caregivers of COVID-19 patients. American Journal of Infection Control. 2020; 48(6): 592-598.

[6] Banerjee D, The COVID-19 Outbreak: crucial role the psychiatrists can play. Asian Journal of Psychiatry. 2020; 50: 102014.

[7] World Health Organization. Mental health and psychosocial considerations during COVID-19 outbreak. 2020.

[8] Braun V, Clarke V, Using thematic analysis in psychology. Qualitative Research in Psychology. 2006; 3: 77-101.

[9] Shaban RZ, Nahidi S, Castillo CS, Li C, Gilroy N, O'Sullivan MVN, et al. SARS-CoV-2 infection and Covid -19. The lived experience and perceptions of patients in isolation and care in an Australian healthcare setting. American journal of Infection Control. 2020.

[10] Beusekom MV, Trust in COVID info sources varies by demographics, beliefs. Centre for Infectious Disease Research and Policy. University of Minnesota. [Internet] [cited on 2021 June 2]

[11] Mansoor T, Mansoor S, Zubair U, Surviving COVID-19: illness narratives of patients and family members in Pakistan. Annals of King Edward Medical University. 2020; 26 (special issue): 157-164.

[12] Jesmi AA, Mohammadzade-Tabrizi Z, Rad M, Hosseinzadeh-Younesi E, Pourhabib A. Lived Experiences of patients with COVID-19 Infection: A phenomenology study. COVID-19 Global Literature on Coronavirus Disease. 2021; 18(1).

[13] Lopez-Leon S, Wegman-Ostrosky T, Perelman C, Sepulveda R, Rebolledo PA, Cuapio AA, et al. More than 50 long-term effects of COVID-19: a systematic review and meta-analysis. Scientific Reports. 2021; $11: 16144$

[14] Klein WM, Weinstein ND. Social comparison and unrealistic optimism about personal risk. Health, coping and wellbeing: Perspectives from social comparison theory. 1997; 25-61

[15] Venema TAG, Pfattheicher S, Perceived susceptibility to COVID-19 infection and narcissistic traits. Personality and Individual Differences. $2021 ; 175$.

[16] Nuriddin A, Jalloh MF, Meyer E, Bunnell R, Bio FA, Jalloh MB, Trust, fear, stigma and disruptions: community perceptions and experiences during periods of low but ongoing transmission of Ebola virus disease in Sierra Leone 2015. BMJ Global Health. 2018; 3(2): e000410.

[17] Serafini G, Parmigiani B, Amerio A, Aguglia A, Sher L, Amore M, The Psychological impact of COVID-19 on mental health in the general population. An International Journal of Medicine. 2020; 529-535.

[18] Reynolds DL, Garay JR, Deamond SL, Moran MK, Gold W, Styra R, Understanding compliance and psychological impact of the SARS quarantine experience, Epidemiology Infection. 2008; 136: 997-1007.

[19] Khan S, Siddique R, Li H, Ali A, Shereen MA, Bashir N, et al. Impact of coronavirus outbreak on psychological health. Journal of Global Health. 2020; 10: 010331.

[20] Jeong H, Yim HW, Song YJ, Ki M, Min JA, Cho J, et al. Mental health status of people isolated due to Middle East respiratory syndrome. Epidemiology Health. 2016; 38: e2016048.

[21] Braunack-Mayer A, Tooher R, Collins JE, Street JM, Marshall H, Understanding the school community's response to school closures during the $2009 \mathrm{H} 1 \mathrm{~N} 1$ influenza pandemic. BMC Public Health. 2013; 13: 344 .

[22] Elshafeey F, Magdi R, Hindi N, Elshebiny M, Farrag N, Mahdy S, et al. A systematic scoping review of COVID-19 during pregnancy and childbirth. International Federation of Gynaecology and Obstetrics. 2020; 150: 47-52.

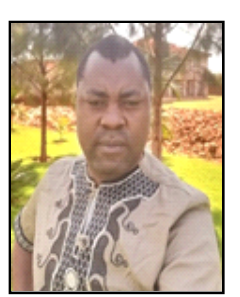

J. Munsaka was born in Binga district of Zimbabwe in 1982. Attained a Diploma in General Nursing and Midwifery from Hwange in 2007 and Harare in 2011 respectively. He also attained a $\mathrm{BSc}$ in Nursing Education from Bindura University, Zimbabwe in 2015. He has just completed the MSc in Nursing Science programme majoring in Maternal and Child Health and Midwifery with University of Zimbabwe 2021.

He has worked as a Clinical Instructor (2008 to 2015) and Nurse Tutor (2015 to date) at Hwange Colliery School of Nursing, Zimbabwe.

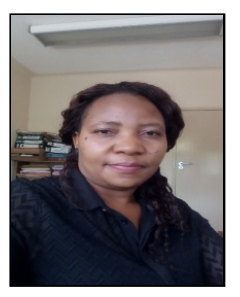

J. Rukweza was born in Nyanga District in 1975. She obtained her first degree in Nursing with the University of Zimbabwe in 2002 . She practiced as a medical-surgical nurse from 2002 to 2006. She then attained a Master degree in Nursing in 2007. She then worked as a Nurse Tutor for 5 years.

She was awarded a Doctorate in Nursing in 2020. She currently works as a Senior Lecturer for the University of Zimbabwe. 